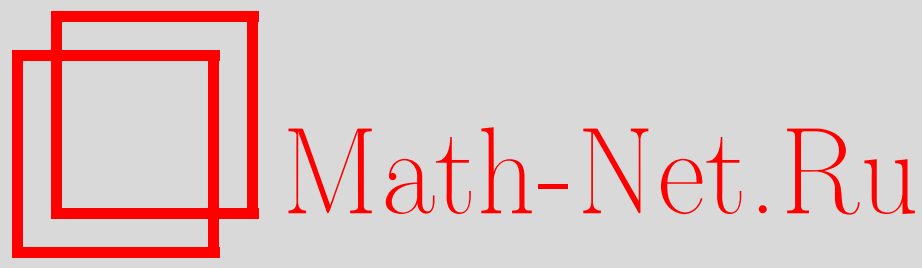

А. Мирджалили, М. Таки, Некоммутативная поправка к потенциалу Корнелла в атомах тяжелого кваркония, TMФ, 2016, том 186, номер 2, 323-329

DOI: https://doi.org/10.4213/tmf8871

Использование Общероссийского математического портала Math-Net.Ru подразумевает, что вы прочитали и согласны с пользовательским соглашением http://www.mathnet.ru/rus/agreement

Параметры загрузки:

IP: 52.6 .47 .48

26 апреля 2023 г., 17:34:31

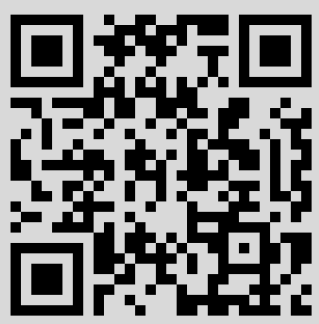




\title{
НЕКОММУТАТИВНАЯ ПОПРАВКА К ПОТЕНЦИАЛУ КОРНЕЛЛА В АТОМАХ ТЯЖЕЛОГО КВАРКОНИЯ
}

\begin{abstract}
Изучается воздействие некоммутативности пространства-времени на потенциал Корнелла в системах тяжелых кваркониев. Известно, что некоммутативность пространства-времени может приводить к возникновению связанных состояний, поэтому в моделях кваркония некоммутативная геометрия пространства-времени рассматривается как поправка. Данные сверхтонких измерений основного состояния боттомония использованы как верхний предел некоммутативной энергетической поправки и выведено максимальное возможное значение параметра некоммутативности $\theta$, а именно $\theta \leqslant 37.94 \cdot 10^{-34} \mathrm{M}^{2}$. Предложенная модель используется для вычисления максимального значения некоммутативной поправки к энергиям чармония и боттомония на уровнях $1 \mathrm{~S}$ и $2 \mathrm{~S}$. Энергетическая поправка как эффект связывания в системе кваркониев меньше для атома чармония, чем для атома боттомония, что соответствует ожиданиям.
\end{abstract}

Ключевые слова: некоммутативное пространство-время, параметр некоммутативности, потенциал Корнелла, тяжелый кварконий, сверхтонкое расщепление.

DOI: $10.4213 / \operatorname{tmf} 8871$

\section{1. ВВЕДЕНИЕ}

Тяжелый кварконий может быть использован для описания нерелятивистского цветного синглетного связанного состояния пары кварк-антикварк, и эта модель очень похожа на нерелятивистские модели атома водорода. Разница между кварконием и атомом водорода заключается в наличии линейного потенциала типа конфайнмента, который прибавляется к кулоновскому потенциалу. В то время как кулоновский потенциал преобладает на относительно коротких расстояниях, потенциал конфайнмента доминирует на длинных расстояниях, что обеспечивает требование конфайнмента кварков. Комбинация этих двух потенциалов называется потенциалом Корнелла [1], [2].

После открытия частицы $J / \psi$ в 1974 г. [3]-[6] развитие моделей кваркония могло бы принести успех при объяснении систематических особенностей тяжелого кваркония вроде $c \bar{c}$ - и $b \bar{b}$-спектров и мод их распада [7]. На протяжении многих лет

* Physics Department, Yazd University, Yazd, Iran.

E-mail: a.mirjalili@yazd.ac.ir, metaki@stu.yazd.ac.ir 
для согласования предсказаний модели с экспериментальными данными разнообразные поправки (релятивистские, спин-спин поправки, однопетлевые и т. д.) вводились в модели простого водородоподобного атома, например в модель кваркония [8]-[15].

Известно, что некоммутативность пространства-времени может приводить к появлению связанных состояний [16], так что мы можем рассматривать геометрию пространства-времени как поправку к моделям кваркония. В настоящей статье мы рассматриваем тяжелый кварконий в некоммутативном (HK) пространстве-времени и используем обычную теорию возмущений для вычисления главной поправки к энергетическим уровням.

Статья организована следующим образом. В разделе 2 мы приводим обзор определений и понятий, необходимых для надлежащего описания НК-геометрии. В разделе 3 мы вводим потенциал Корнелла в НК-пространстве-времени. В разделе 4, используя теорию возмущений, мы получаем общий вид НК-поправки к энергии для различных уровней энергии тяжелого кваркония на коротких расстояниях. В разделе 5 мы рассматриваем экспериментальное значение сверхтонкой энергетической поправки к спектру для состояния $1 \mathrm{~S}$ и выводим верхний предел энергетической $\mathrm{HK}$ поправки. Это позволяет нам оценить максимальное значение параметра $\theta$, который присутствует в коммутационном соотношении. Затем мы определяем максимальное значение энергетической $\mathrm{HK}$-поправки для уровней $1 \mathrm{~S}$ и $2 \mathrm{~S}$ чармония и боттомония. Наконец, раздел 6 содержит выводы.

\section{2. ОСНОВНЫЕ ОПРЕДЕЛЕНИЯ}

НК-алгебра определяется в терминах операторов $\hat{x}^{i}, i=1, \ldots, n$, координат пространства-времени, которые удовлетворяют коммутационным соотношениям

$$
\left[\hat{x}^{i}, \hat{x}^{j}\right]=\mathrm{i} \hbar \theta \hat{\omega}^{i j}(\hat{x})
$$

где $\hat{\omega}^{i j}(\hat{x})$ - антисимметричный тензорный оператор, описывающий некоммутативность пространства-времени, и $\theta$ - параметр некоммутативности. Величина $\hbar \theta \hat{\omega}^{i j}(\hat{x})$ как постоянный параметр некоммутативности является действительной и имеет размерность площади. Кроме того, можно построить новую теорию поля в НК-пространстве-времени, если заменить стандартное произведение полей на звездочное [13]:

$$
\begin{aligned}
& (f * g)(x)=f(\hat{x}) g(x)=f g+\frac{\mathrm{i} \theta}{2} \partial_{i} f \omega^{i j} \partial_{j} g- \\
& \quad-\frac{\theta^{2}}{4}\left[\frac{1}{2} \omega^{i j} \omega^{k l} \partial_{i} \partial_{k} f \partial_{j} \partial_{l} g-\frac{1}{3} \omega^{i j} \partial_{j} \omega^{k l}\left(\partial_{i} \partial_{k} f \partial_{l} g-\partial_{k} f \partial_{i} \partial_{l} g\right)\right]+O\left(\theta^{3}\right),
\end{aligned}
$$

где

$$
\hat{x}^{i}=x^{i}+\frac{\mathrm{i} \theta}{2} \omega^{i j} \partial_{j}+O\left(\theta^{2}\right) .
$$

Общепринятым является выбор $\hat{\omega}^{i j}(\hat{x})=\left(\begin{array}{rr}0 & 1 \\ -1 & 0\end{array}\right)$, однако было доказано, что этот выбор нарушает вращательную симметрию кулоновского потенциала в водородоподобных моделях и снимает вырождение энергетических уровней [17], [18], что нежелательно. На самом деле эти особенности не должны иметь место, так как природа водородного атома определена экспериментально. Поэтому мы ищем альтернативный выбор: выбираем $\hat{\omega}^{i j}(\hat{x})$ так, чтобы это не нарушило вращательную 
симметрию атома кваркония. Затем мы рассчитываем поправку, которая связана с некоммутативностью пространства-времени, для энергетических уровней тяжелого кваркония.

\section{3. ПОТЕНЦИАЛ КОРНЕЛЛА В НК-ПРОСТРАНСТВЕ-ВРЕМЕНИ}

Потенциал Корнелла часто выбирается в качестве потенциала взаимодействия между тяжелым кварком и антикварком в модели кваркония [8]. Потенциал Корнелла имеет вид

$$
V(r)=-\frac{4}{3} \frac{\hbar c \alpha_{\mathrm{s}}}{r}+k r
$$

где $\alpha_{\mathrm{s}}-$ константа сильной связи и $k$ - постоянная конфайнмента. Первый член в потенциале получается за счет одноглюонного пертурбативного обмена и преобладает во взаимодействиях на коротких расстояниях, в то время как второй член обеспечивает конфайнмент кварков. Для построения НК-потенциала Корнелла мы заменим расстояние $r$ в соотношении (4) на оператор $\hat{r}$, который определяется как

$$
\begin{aligned}
\hat{r}=\sqrt{\hat{x}^{i} \hat{x}_{i}} & =\sqrt{r^{2}+\left(\frac{\mathrm{i} \theta}{2} x^{i} \omega_{i j} \partial^{j}-\frac{\theta^{2}}{4} \omega^{i j} \partial_{j} \omega_{i k} \partial^{k}+O\left(\theta^{3}\right)\right)} \approx \\
& \approx r+\frac{1}{r}\left(\frac{\mathrm{i} \theta}{4} x^{i} \omega_{i j} \partial^{j}-\frac{\theta^{2}}{8} \omega^{i j} \partial_{j} \omega_{i k} \partial^{k}\right),
\end{aligned}
$$

при этом

$$
\begin{aligned}
\frac{1}{\hat{r}}=\frac{1}{\sqrt{\hat{x}^{i} \hat{x}_{i}}} & =\left[r^{2}+\left(\frac{\mathrm{i} \theta}{2} x^{i} \omega_{i j} \partial^{j}-\frac{\theta^{2}}{4} \omega^{i j} \partial_{j} \omega_{i k} \partial^{k}+O\left(\theta^{3}\right)\right)\right]^{-1 / 2} \approx \\
& \approx \frac{1}{r}+\frac{1}{r^{3}}\left(-\frac{\mathrm{i} \theta}{4} x^{i} \omega_{i j} \partial^{j}+\frac{\theta^{2}}{8} \omega^{i j} \partial_{j} \omega_{i k} \partial^{k}\right),
\end{aligned}
$$

где мы использовали равенство (3), чтобы заменить $\hat{x}^{i}$ подходящим выражением. Теперь НК-потенциал Корнелла $V_{\text {NC }}(\hat{r})$ записывается следующим образом:

$$
V_{\mathrm{NC}}(\hat{r})=-\frac{4}{3} \frac{\hbar c \alpha_{\mathrm{s}}}{\hat{r}}+k \hat{r}
$$

Из формул (5)-(7), очевидно, следует, что на коротких расстояниях линейное слагаемое $k \hat{r}$ пренебрежимо мало по сравнению с кулоноподобным членом, и мы можем записать НК-потенциал на коротких расстояниях как

$$
V_{\mathrm{NC}}(\hat{r}) \approx-\frac{4}{3} \frac{\hbar c \alpha_{\mathrm{s}}}{\hat{r}}=V(r)+\Delta V_{\mathrm{NC}}
$$

где $V(r)=-(4 / 3) \hbar c \alpha_{\mathrm{s}} / r$, что подобно кулоновскому потенциалу в атоме водорода, и $\Delta V_{\mathrm{NC}}-$ поправочный член, связанный с НK-геометрией пространства-времени.

\section{4. ЭНЕРГЕТИЧЕСКАЯ ПОПРАВКА ДЛЯ НК-ПРОСТРАНСТВА-ВРЕМЕНИ}

Теперь мы можем использовать обычную теорию возмущений для вычисления главной поправки к уровням энергии:

$$
\Delta E_{\mathrm{NC}}=\left\langle\psi^{0}\left|\Delta V_{\mathrm{NC}}\right| \psi^{0}\right\rangle
$$


где $\psi^{0}$ - невозмущенная волновая функция атома водорода, $\left|\psi^{0}\right\rangle=R_{n l}(r) Y_{l}^{m}(\theta, \phi)$, но радиус Бора $a_{0}$ должен быть заменен на радиус кваркония $a_{0}^{\prime}$, который определяется как

$$
a_{0}^{\prime}=\frac{\hbar}{\mu_{\mathrm{q}} c \alpha_{\mathrm{s}}},
$$

где $\mu_{\mathrm{q}}$ - приведенная масса покоя в тяжелом кварконии $c \bar{c}$ или $b \bar{b}$ (т. е. $m_{c} / 2$ или $\left.m_{b} / 2\right)$ и $\alpha_{\mathrm{s}}-$ соответствующая константа сильной связи.

Теперь последний шаг для получения общей формы энергетической HK-поправки сводится к определению вида параметра некоммутативности $\hat{\omega}^{i j}(\hat{x})$. Как уже упоминалось, общепринятый вид $\hat{\omega}^{i j}(\hat{x})=\left(\begin{array}{rr}0 & 1 \\ -1 & 0\end{array}\right)$ нарушает вращательную симметрию HK-потенциала в $(8)$, так как $\left[L_{i}, V_{\mathrm{NC}}(\hat{r})\right] \neq 0$, где $L_{i}=-\mathrm{i} \hbar \varepsilon^{i j k} x_{j} \partial_{k}$ - оператор момента импульса. Однако можно выбрать $\hat{\omega}^{i j}(\hat{x})$ так, чтобы сохранить вращательную симметрию системы. Один из подходящих выборов [18] таков:

$$
\hat{\omega}^{i j}=\varepsilon^{i j k} x_{k} f(r),
$$

где $f(r)$ - вспомогательная функция. При этом соответствующая НК-алгебра записывается как

$$
\left[\hat{x}^{i}, \hat{x}^{j}\right]=\mathrm{i} \hbar \theta \varepsilon^{i j k} \hat{x}_{k} f(\hat{r}) .
$$

Если подставить выражение (11) в формулу (6), то слагаемое $1 / \hat{r}$ в потенциале принимает вид

$$
\frac{1}{\hat{r}} \approx \frac{1}{r}+\frac{1}{r^{3}}\left(-\frac{\mathrm{i} \theta}{4 \hbar} x_{i} \varepsilon^{i j k} x_{k} f(r) \partial_{j}+\frac{\theta^{2} L^{2} f^{2}(r)}{8 \hbar^{2}}\right) .
$$

Очевидно, член $x_{i} \varepsilon^{i j k} x_{k} \partial_{j}$ в этом соотношении равен нулю $(A \cdot(A \times B)=0)$, и мы получаем

$$
\frac{1}{\hat{r}} \approx \frac{1}{r}+\frac{1}{r^{3}}\left(\frac{\theta^{2} L^{2} f^{2}(r)}{8 \hbar^{2}}\right) .
$$

Отсюда следует, что сохранение вращательной симметрии теперь обеспечено:

$$
\left[L_{i}, V_{\mathrm{NC}}(\hat{r})\right]=0 .
$$

Итак, для потенциала Корнелла на коротких расстояниях ведущий порядок теории возмущений, требующихся в НК-теории, имеет вид

$$
\Delta V_{\mathrm{NC}}=-\frac{\hbar c \alpha_{\mathrm{s}}}{r^{3}}\left(\frac{\theta^{2} L^{2} f^{2}(r)}{6 \hbar^{2}}\right) .
$$

Подходящим выбором является $f(r)=1 / r$. Это дает параметр $\theta$, имеющий размерность площади, который соотносится с НK-теорией. При таком выборе мы имеем

$$
\Delta V_{\mathrm{NC}}=-\frac{c \alpha_{\mathrm{s}} \theta^{2} L^{2}}{6 \hbar r^{5}}
$$

Теперь из формулы (9) получается поправка к энергетическим уровням, соответствующая НК-геометрии пространства-времени:

$$
\Delta E_{\mathrm{NC}}=-\frac{\hbar c \alpha_{\mathrm{s}} \theta^{2} l(l+1)}{6}\left\langle\frac{1}{r^{5}}\right\rangle
$$

где $l$ - азимутальное квантовое число. 
Используя волновую функцию атома водорода $\left|\psi^{0}\right\rangle=R_{n l}(r) Y_{l}^{m}(\theta, \phi)$, мы получаем среднее значение для $1 / r^{5}$ :

$$
\begin{aligned}
\left\langle\frac{1}{r^{5}}\right\rangle= & \frac{1}{\left(a_{0}^{\prime}\right)^{5} n^{5}} \frac{1}{l(l+1 / 2)(l+1)(l+3 / 2)(l+2)(l-1)(l-1 / 2)} \times \\
& \times\left(\frac{5}{6}\left(3 n^{2}-l(l+1)\right)-\left(l+\frac{3}{2}\right)\left(l-\frac{1}{2}\right)\right) .
\end{aligned}
$$

Таким образом, общий вид поправочного члена для энергетических уровней записывается как

$$
\begin{aligned}
\Delta E_{\mathrm{NC}}= & -\frac{\alpha_{\mathrm{s}} \theta^{2}}{6\left(a_{0}^{\prime}\right)^{5} n^{5}} \frac{1}{(l+1 / 2)(l+3 / 2)(l+2)(l-1)(l-1 / 2)} \times \\
& \times\left(\frac{5}{6}\left(3 n^{2}-l(l+1)\right)-\left(l+\frac{3}{2}\right)\left(l-\frac{1}{2}\right)\right) .
\end{aligned}
$$

Следует заметить, что величина $\Delta E_{\mathrm{NC}}$ расходится при $l=1$ вследствие сингулярности $\left\langle 1 / r^{5}\right\rangle$. На самом деле в работе [19] было показано, что в НК-квантовой электродинамике разложение по параметру $\theta$, которое используется для оценки поправки к энергетическим уровням в модели кваркония, не является перенормируемым. С другой стороны, заметим, что состояние $l=1$ не используется в наших расчетах. Для малых расстояний, которые описывают уровни $1 \mathrm{~S}$ и $2 \mathrm{~S}$, мы не столкнулись ни с какими сингулярностями. Один из способов преодолеть трудности, возникающие для состояния $l=1$, состоит в рассмотрении потенциала конфайнмента $k r$.

\section{5. СВЕРХТОНКАЯ ПОПРАВКА}

НК-поправка к энергии тяжелого кваркония, полученная в соотношении (20), является функцией параметра некоммутатитвности $\theta$. Величину этого параметра нельзя получить из экспериментальных данных, поэтому мы принимаем во внимание сверхтонкие измерения, т. е. поправку к наинизшему энергетическому уровню для модели кваркония, в качестве верхнего предела энергетической HK-поправки и получаем максимально возможное значение $\theta$. Можно ожидать, что некоммутативность пространства-времени проявится в очень небольших смещениях в пространстве-времени с очень высокой плотностью энергии. Таким образом, подходящим кандидатом может быть изучение переходов боттомония. Согласно данным из работы [20] среднее значение сверхтонкого расщепления основного состояния боттомония $\eta_{b}(1 \mathrm{~S})$ имеет вид

$$
\Delta M_{\mathrm{hf}}(1 \mathrm{~S})_{b \bar{b}}=M(\Upsilon(1 \mathrm{~S}))-M\left(\eta_{b}(1 \mathrm{~S})\right)=64.1 \pm 2.0 \mathrm{M \ni} \mathrm{B} / c^{2} .
$$

Используя этот верхний предел, подставляя выражение (10) в формулу (20) и полагая $n=1, l=0$, мы получаем

$$
\Delta E_{\mathrm{NC}} \leqslant \Delta M_{\mathrm{hf}}(1 \mathrm{~S})_{b \bar{b}}, \quad \frac{13 \alpha_{\mathrm{s}}^{6} m_{c}^{5} c^{6}}{16 \hbar^{4}} \theta^{2} \leqslant 64.1 \mathrm{M \ni B.}
$$

Для $m_{c}=1.55$ ГэВ $/ c^{2}$ и $\alpha_{\mathrm{s}}=0.416$ [13] мы имеем

$$
\theta \leqslant 37.94 \cdot 10^{-34} \mathrm{M}^{2},
$$

что соответствует расстоянию $\sqrt{\theta} \leqslant 0.61 \cdot 10^{-16} \mathrm{M}$. 
Используя полученное значение $\theta$, мы можем найти максимальную поправку для уровня $2 \mathrm{~S}$ в боттомонии, связанную с некоммутативностью геометрии:

$$
\Delta E_{\mathrm{NC}}(2 \mathrm{~S}) \leqslant 3.6 \mathrm{M \ni B}
$$

где экспериментальный результат для сверхтонкого расщепления имеет вид

$$
\Delta M_{\mathrm{hf}}(2 \mathrm{~S})_{b \bar{b}}=M(\Upsilon(2 \mathrm{~S}))-M\left(\eta_{b}(2 \mathrm{~S})\right)=48.7 \pm 2.7 \mathrm{M \ni B} / c^{2} .
$$

Поправочные слагаемые, связанные с некоммутативностью, для уровней $1 \mathrm{~S}$ и $2 \mathrm{~S}$ чармония и боттомония приведены в табл. 1.

ТАБлицА 1. Некоммутативные поправочные данные к энергии для уров-

\begin{tabular}{|c|c|c|}
\hline & $\begin{array}{l}\text { Экспериментальная энергия } \\
\text { сверхтонкого расщепления }\end{array}$ & $\begin{array}{c}\text { Некоммутативная } \\
\text { энергетическая поправка }\end{array}$ \\
\hline$\eta_{c}(1 \mathrm{~S})$ & $\begin{aligned} \Delta M_{\mathrm{hf}}(1 \mathrm{~S})_{c \bar{c}} & =M(J / \psi(1 \mathrm{~S}))-M\left(\eta_{c}(1 \mathrm{~S})\right)= \\
& =116.6 \pm 1.2 \mathrm{M \ni B} / c^{2}\end{aligned}$ & $\Delta E_{\mathrm{NC}}(1 \mathrm{~S}) \leqslant 195$ кэВ \\
\hline$\eta_{c}(2 \mathrm{~S})$ & $\begin{aligned} \Delta M_{\mathrm{hf}}(2 \mathrm{~S})_{c \bar{c}} & =M(\psi(2 \mathrm{~S}))-M\left(\eta_{c}(2 \mathrm{~S})\right)= \\
& =49 \pm 4 \mathrm{M \ni B} / c^{2}\end{aligned}$ & $\Delta E_{\mathrm{NC}}(2 \mathrm{~S}) \leqslant 11 \mathrm{\kappa \ni B}$ \\
\hline$\eta_{b}(1 \mathrm{~S})$ & $\begin{aligned} \Delta M_{\mathrm{hf}}(1 \mathrm{~S})_{b \bar{b}} & =M(\Upsilon(1 \mathrm{~S}))-M\left(\eta_{b}(1 \mathrm{~S})\right)= \\
& =64.1 \pm 2.0 \mathrm{M \ni B} / c^{2}\end{aligned}$ & $\Delta E_{\mathrm{NC}}(2 \mathrm{~S}) \leqslant 64.1 \mathrm{M \ni B}$ \\
\hline$\eta_{b}(2 \mathrm{~S})$ & $\begin{aligned} \Delta M_{\mathrm{hf}}(2 \mathrm{~S})_{b \bar{b}} & =M(\Upsilon(2 \mathrm{~S}))-M\left(\eta_{b}(2 \mathrm{~S})\right)= \\
& =48.7 \pm 2.7 \mathrm{M \ni B} / c^{2}\end{aligned}$ & $\Delta E_{\mathrm{NC}}(2 \mathrm{~S}) \leqslant 3.6 \mathrm{M \ni B}$ \\
\hline
\end{tabular}
ней $1 \mathrm{~S}$ и $2 \mathrm{~S}$ систем тяжелых кваркониев.

\section{6. ЗАКЛЮЧЕНИЕ}

Мы изучили влияние некоммутативности пространства-времени на энергетические уровни нерелятивистских тяжелых кваркониев $c \bar{c}$ и $b \bar{b}$. Хотя мы рассмотрели только потенциал кулоновского типа, в НК-геометрии мы получили поправку для этого потенциала, которая описывает эффект конфайнмента в системе кваркониев. Так как точное значение параметра некоммутативности $\theta$ неизвестно, мы взяли верхний предел этого значения. Сверхтонкое расщепление основного состояния боттомония, которое происходит в очень малой области пространства-времени, может быть хорошим приближением для поправки к верхнему энергетическому уровню, вызванной некоммутативностью пространства-времени. Мы использовали этот верхний предел и оценили максимальное значение параметра некоммутативности: $\theta \leqslant 37.94 \cdot 10^{-34}$ м. Используя это значение для $\theta$, мы оценили максимальную поправку к энергии состояний $1 \mathrm{~S}$ и $2 \mathrm{~S}$ систем тяжелых кваркониев $c \bar{c}$ и $b \bar{b}$. Так как атом чармония легче, чем атом боттомония, энергетическая поправка меньше для атома $c \bar{c}$, чем для атома $b \bar{b}$.

Применение НК-геометрии для внесения поправок в вершину фотон-кварк в глубоком неупругом рассеянии лептона на нуклонах нам представляется весьма плодотворным, о чем мы надеемся написать в ближайшем будущем. С другой стороны, 
так как уравнение Шредингера с потенциалом Корнелла может рассматриваться как частный случай биконфлюентного уравнения Гойна [21]-[25], было бы интересно в дальнейшем провести изучение этого потенциала в НК-геометрии, используя дифференциальное уравнение Гойна, которое ведет себя более гладко, имея меньше особенностей.

\section{Список литературы}

[1] E. Eichten, K. Gottfried, T. Kinoshita, K. D. Lane, T. M. Yan, Phys. Rev. D, 17:11 (1978), 3090-3117.

[2] E. Eichten, K. Gottfried, T. Kinoshita, K. D. Lane, T. M. Yan, Phys. Rev. D, 21:1 (1980), 203-233.

[3] J. J. Aubert, U. Becker, P. J. Biggs et al., Phys. Rev. Lett., 33:23 (1974), 1404-1406.

[4] J.-E. Augustin, A. M. Boyarski, M. Breidenbach et al., Phys. Rev. Lett., 33:23 (1974), 1406-1408.

[5] C. Bacci, R. B. Celio, M. Berna-Rodini et al., Phys. Rev. Lett., 33:23 (1974), 1408-1410.

[6] G.S. Abrams, D. Briggs, W. Chinowsky et al., Phys. Rev. Lett., 33:24 (1974), 1453-1455.

[7] N. Rambilla, M. Krämer, R. Mussa et al., Heavy quarkonium physics, CERN Yellow Report, CERN, Geneva, 2005, arXiv: hep-ph/0412158.

[8] E. Eichten, K. Gottfried, T. Kinoshita, J. Kogut, K. D. Lane, T.-M. Yan, Phys. Rev. Lett., 34:6 (1975), 369-372.

[9] H. J. Schnitzer, Phys. Rev. Lett., 35:22 (1975), 1540-1543.

[10] S. N. Gupta, S. F. Radford, W. W. Repko, Phys. Rev. D, 26:11 (1982), 3305-3308.

[11] S. N. Gupta, S. F. Radford, W. W. Repko, Phys. Rev. D, 31:1 (1985), 160-163.

[12] S. N. Gupta, S. F. Radford, W. W. Repko, Phys. Rev. D, 34:1 (1986), 201-206.

[13] S. F. Radford, W. W. Repko, Phys. Rev. D, 75:7 (2007), 074031.

[14] S. F. Radford, W. W. Repko, Nucl. Phys. A, 865:1 (2011), 69-75.

[15] T. J. Burns, Phys. Rev. D, 87:3 (2013), 034022, 8 pp.

[16] D. V. Vassilevich, A. Yurov, Phys. Rev. D, 69:10 (2004), 105006, 5 pp.

[17] M. Chaichian, M. M. Sheikh-Jabbari, A. Tureanu, Phys. Rev. Lett., 86:13 (2001), 2716-2719.

[18] V. G. Kupriyanov, J. Phys. A: Math. Theor., 46:24 (2013), 245303, 7 pp., arXiv: 1209.6105.

[19] R. Wulkenhaar, JHEP, 03 (2002), 024, 35 pp., arXiv: hep-th/0112248.

[20] J. Beringer, J.-F. Arguin, R. M. Barnett et al. [Particle Data Group], Phys. Rev. D, 86:1 (2012), 010001, 1528 pp.

[21] С. Славянов, В. Лай, Специальные функиии: единая теория, основанная на анализе особенностей, Невский Диалект, СПб., 2002.

[22] С. Ю. Славянов, ТМФ, 119:1 (1999), 3-19.

[23] M. Hortacsu, "Heun functions and their uses in physics", Proceedings of the 13th Regional Conference on Mathematical Physics (Antalya, Turkey, October 27-31, 2010), eds. U. Camci, I. Semiz, World Sci., Singapore, 2013, 23-39, arXiv: 1101.0471.

[24] В. Лай, ТМФ, 101:3 (1994), 360-368.

[25] С. Ю. Славянов, ТМФ, 182:2 (2015), 223-230.

Поступила в редакцию 11.02.2015, после доработки 25.03.2015 